\title{
Macroamilasemia en el Hospital Víctor Lazarte Echegaray de Trujillo, Perú: reporte de un caso
}

\section{Macroamylasemia at the Hospital Víctor Lazarte Echegaray in Trujillo (Peru): Case report}

Sandro Ruíz-García. * 이

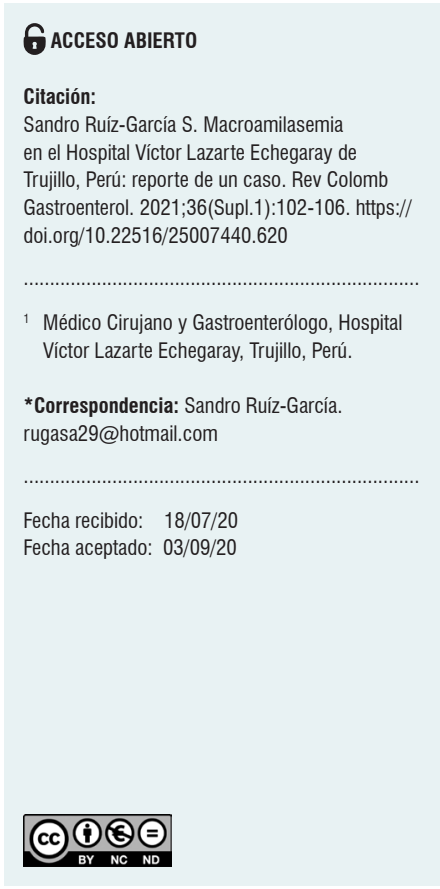

\begin{abstract}
Resumen
La macroamilasemia debe sospecharse en todo paciente con aumento catalítico de amilasa a plasmática persistente y sin clínica de dolor abdominal, descartando otras etiologías de patología pancreáticas y extrapancreática. La macroamilasemia se caracteriza por la unión de complejos de amilasa a con inmunoglobulina, más frecuente inmunoglobulina $A$; pueden presentarse 3 tipos de macroamilasemia. Es importante realizar el diagnóstico diferencial de otras patologías que puedan causar el aumento de la amilasa y así evitar los procedimientos innecesarios. Se presenta el caso de un paciente de 53 años, de sexo femenino, que ingresó a emergencia por clínica de dolor abdominal y hiperamilasemia, que fue diagnosticada inicialmente de pancreatitis aguda.
\end{abstract}

\section{Palabras clave}

Hiperamilasemia, macroamilasemia, pancreatitis aguda.

\begin{abstract}
Macroamylasemia should be suspected in any patient with a persistent catalytic increase of plasma a-amylase but no other clinical signs of abdominal pain after ruling out other causes of pancreatic and extra-pancreatic disease. The binding of a-amylase complexes with immunoglobulin, most commonly immunoglobulin $\mathrm{A}$, characterizes this condition. Macroamylasemia is classified into three kinds. To prevent unnecessary procedures, it is critical to make a differential diagnosis of other conditions that can cause amylase increase. The present article reports the case of a 53-year-old female patient who was admitted to the emergency room with abdominal pain and hyperamylasemia, who was initially diagnosed with acute pancreatitis.
\end{abstract}

\section{Keywords}

Macroamylasemia; Hyperamylasemia; Acute pancreatitis.

\section{INTRODUCCIÓN}

El reto del diagnóstico de la macroamilasemia se presenta cuando se asocia a dolor abdominal, que puede dar un falso diagnóstico de pancreatitis aguda. La pancreatitis aguda se caracteriza por dolor abdominal tipo pancreático, un estudio de imagen con evidencia de pancreatitis y bioquímicamente por un aumento de tres veces el límite superior normal plasmático de la concentración catalítica de las enzimas amilasa $a$ y lipasa (1-4).
Sin embargo, este aumento también puede estar relacionado con enfermedades metabólicas pancreáticas y extrapancreáticas y anormalidades de los conductos pancreáticos (5). Las principales causas de hiperamilasemia no pancreática son las enfermedades de las glándulas salivares (parotiditis, litiasis), las enfermedades intestinales (infarto intestinal, enfermedad celíaca, colitis ulcerosa y peritonitis), las enfermedades hepáticas crónicas, las neoplasias, la cetoacidosis diabética, la insuficiencia renal, el embarazo ectópico, el ovario poliquístico, el traumatismo craneal y la 
macroamilasemia (6-8). La existencia de la macroamilasa fue descrita inicialmente por Wilding, en el año de 1964 (6). El término fue propuesto por Berk en 1967 y publicado en reportes de casos (9).

La macroamilasemia, una condición rara, pero benigna, se caracteriza por hiperamilasemia o elevación de los niveles de amilasa $\alpha$ sérica sin elevación de la amilasa $\alpha$ en orina, siempre y cuando la función renal esté preservada (10). La macroamilasemia se puede encontrar en el 0,1\%-1,5\% de la población general no alcohólica; las isoformas de la amilasa están codificadas en el cromosoma 1p21 (11). Es más común en hombres que en mujeres debido a razones desconocidas. La macroamilasemia es una condición en la que la proteína de amilasa sérica se une a otro suero de proteínas como las inmunoglobulinas A ( IgA) (hasta en el 92\%) y $\mathrm{G}(\mathrm{IgG})(<30 \%)$, entre otros ( $<5 \%)$, y forman complejos macromoléculas de gran tamaño, que exceden los $400 \mathrm{KDa}$ (amilasa en forma citoplasmática activa contiene 496 aminoácidos con un peso molecular de $54 \mathrm{KDa}$ ) (11-13).

La macroamilasemia puede diagnosticarse indirectamente midiendo la actividad de amilasa en la orina; más específicamente, la relación de depuración amilasacreatinina (ACR) puede ayudar a aumentar y diferenciar la hiperamilasemia. Esta relación se calcula utilizando la siguiente ecuación:

$$
\begin{gathered}
\mathrm{ACR}=(\text { amilasa }[\text { orina }] \times \text { creatinina }[\text { suero }]) /(\text { amilasa } \\
[\text { suero }] \times \text { creatinina }[\text { orina }]) \times 100
\end{gathered}
$$

Una ACR $>5 \%$ sugiere pancreatitis aguda.

También puede ocurrir un aumento de la ACR en la cetoacidosis diabética, la cirugía y la enfermedad renal. Una ACR $<1 \%$ sugiere macroamilasemia (11). La croma- tografía y la precipitación con polietilenglicol (PEG) son los métodos de elección para detectar las macromoléculas que incluyen macroamilasa $(6,11)$.

\section{PRESENTACIÓN DE CASO CLÍNICO}

Paciente mujer de 53 años que ingresó por un cuadro abdominal agudo tipo pancreático, como antecedente de importancia, hipertensa, en tratamiento con losartán de 50 mg cada 12 horas, y colecistectomizada. Durante el examen físico presentó dolor a la palpación en el epigastrio. Se realizaron los siguientes exámenes complementarios: amilasa, de 1407 U/L; ecografía, con páncreas normal; colédoco, de 7,4 $\mathrm{mm}$; y leve hidronefrosis izquierda. La paciente fue hospitalizada con diagnóstico de pancreatitis aguda leve. A los 5 días se realizó una amilasa de control que mostró un resultado de 1419 U/L (asociado sin dolor). La paciente es dada de alto para estudio de hiperamilasemia por consultorio externo. En la Tabla 1 se resume la evolución de los estudios solicitados durante su estudio en consultorio externo.

Durante el seguimiento del paciente se realizaron exámenes de laboratorio para descartar las patologías asociadas que puedan causar hiperamilasemia (Tabla 1). También se llevó a cabo endoscopia con toma de biopsia de duodeno, que mostró arquitectura glandular asociada a infiltrado crónico inespecífico, conteo de linfocitos intraepiteliales $<40 / 100$ células epiteliales, sin atrofia. En el estudio de colonoscopia se observaron divertículos en colon sigmoides. En cuanto a los estudios de imagen de tomografía del tejido blando del cuello, no hubo linfadenopatías, y la tomografía toracoabdominopélvica no mostró evidencia de cáncer (Figura 1); la colangiorresonancia no evidenció coledocolitiasis, con vía biliar normal

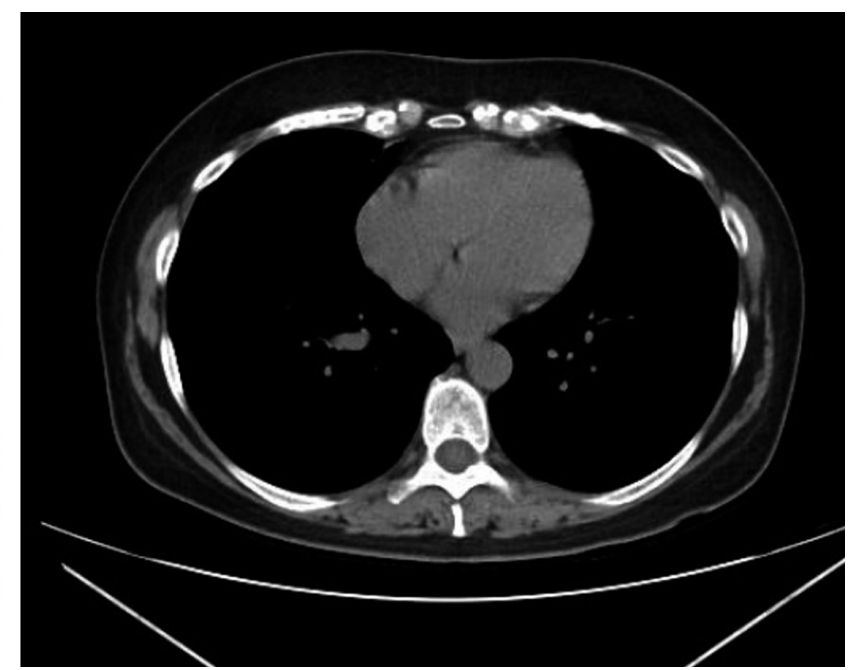

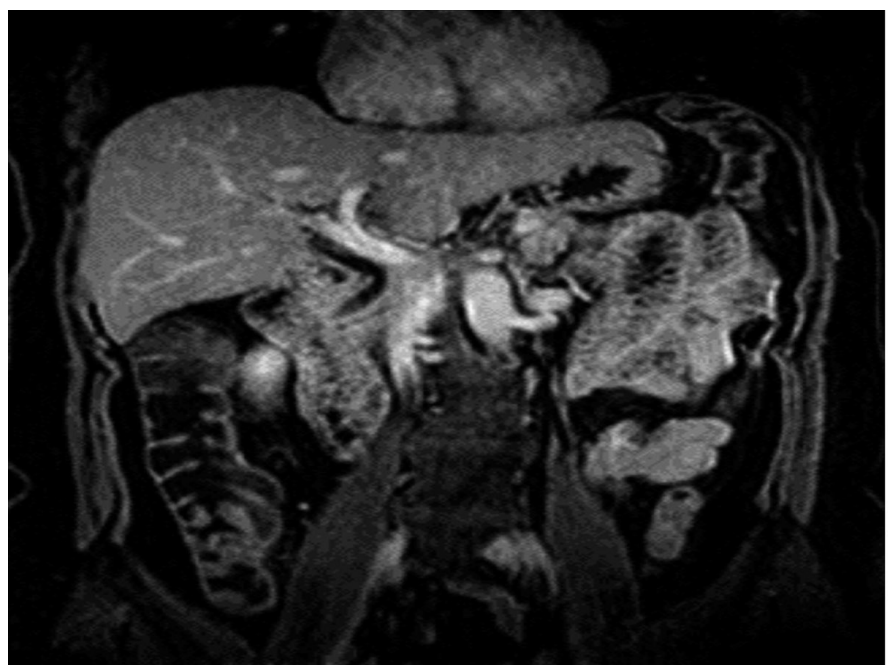

Figura 1. Tomografía abdominopélvica. 
Tabla 1. Resumen de evolución de los exámenes

\begin{tabular}{|c|c|c|c|c|c|c|}
\hline & $\begin{array}{l}\text { Ingreso al } \\
\text { hospital }\end{array}$ & 5 días & 3 meses & 8 meses & 10 meses & 1 año \\
\hline Amilasa a (UI/L) & 1407 & 1419 & 2445 & 2454 & 2495 & 1174 \\
\hline Lipasa (UI/L) & & 32 & & --- & & \\
\hline Creatinina (mg/dL) & 0,48 & --- & 0,54 & -- & --- & --- \\
\hline Urea $(\mathrm{mg} / \mathrm{dL})$ & 23 & --- & 22 & --- & --- & --- \\
\hline Depuración de creatinina (mL/min) & 120 & --- & 107 & --- & --- & --- \\
\hline Glucosa (mg/dL) & 106 & --- & 88 & 90 & --- & --- \\
\hline AST/GOT (UI/L) & 17 & --- & 18 & 22 & --- & --- \\
\hline ALT/GPT (UI/L) & 13 & -- & 10 & 27 & -- & --- \\
\hline Albúmina (g/dL) & 4,50 & --- & 4,54 & 4,43 & --- & --- \\
\hline Proteína total (g/dL) & 7,32 & -- & 7,30 & 7,45 & -- & -- \\
\hline Globulina (g/dL) & 2,82 & --- & 2,76 & 3,02 & -- & --- \\
\hline Fosfatasa alcalina & 200 & -- & 205 & 244 & -- & -- \\
\hline Gammaglutamiltranspeptidasa & 40 & -- & & 14 & -- & -- \\
\hline Bilirrubinas totales (mg/dL) & 0,8 & -- & 0,57 & 0,53 & -- & -- \\
\hline Triglicéridos & 156 & -- & & 66 & -- & -- \\
\hline Hemoglobina (g/dL) & 12,8 & --- & & 12,9 & --- & --- \\
\hline Plaquetas $\mathrm{mL} / \mathrm{mm}^{3}$ & 350.000 & -- & & 380.000 & -- & -- \\
\hline TSH (mUl/dL) & -- & -- & -- & 3,1 & -- & -- \\
\hline $\mathrm{T}_{4}$ libre (ng/dL) & -- & -- & -- & 1,83 & -- & -- \\
\hline ANA & -- & -- & -- & -- & Negativo & --- \\
\hline VRDL & -- & -- & -- & -- & Negativo & -- \\
\hline Factor reumatoideo & -- & --- & -- & -- & 28,18 & -- \\
\hline Hepatitis B, core total & --- & --- & -- & -- & No reactivo & -- \\
\hline Hepatitis B, antígeno australiano & -- & -- & -- & -- & No reactivo & -- \\
\hline Hepatitis C-HCV & --- & --- & -- & --- & No reactivo & -- \\
\hline Elisa para VIH & -- & --- & -- & -- & No reactivo & --- \\
\hline AMA & --- & -- & --- & Negativo & & -- \\
\hline
\end{tabular}

Fuente: Sistema de Atención Historia Clínica Hospital Lazarte Echegaray - Essalud. TGO: transaminasa glutámico-oxalacética (VN: 0-35 UI); TGP: transaminasa glutámico-pirúvica (VN: 0-35 UI); BT: bilirrubina total (VN: 0,3-1,2 mg/dL); BD: bilirrubina directa (VN: 0-0,3 mg/dL); PT: proteínas totales (VN: 6-8 g/dL); ALB: albúmina (VN: 3,5-5,5 g/dL); FA: fosfatasa alcalina (36-129 UI); GGT: gamma-glutamil transpeptidasa (VN: 8-78 UI); amilasa sérica (VN:0-100UI/L); T4 (VN: 0,8 a 1,9 ng/dL); TSH (VN: 0.5-4.5 mU/dL); plaquetas $\left(\mathrm{VN}: 150-450 \mathrm{ml} / \mathrm{mm}^{3}\right)$; hemoglobina (VN: 12-14 g/dL); creatinina sérica (VN: 0,4 a 1,4 mg/dL); VRDL: The Viral and Rickettsial Disease Laboratory; VN: valores normales.

(Figura 2). Para ampliar el estudio de hiperamilasemia se solicitó un estudio de depuración de amilasa (ACR), cuyo resultado fue $0,02 \%$, y un estudio de confirmación de macroamilasemia, que dio positivo (Tabla 2). Durante su evaluación en consultorio externo, la paciente estuvo asintomática.

\section{DISCUSIÓN}

El caso descrito es una paciente de sexo femenino, si se tiene en cuenta que la macroamilasemia es más frecuente en el sexo masculino (11). Esta paciente se caracterizaba porque el dolor abdominal de ingreso se asoció a hipera- 
Tabla 2. Estudio de macroamilasemia

\begin{tabular}{|lc|}
\hline & Resultado \\
\hline Depuración de amilasa & 0,02 \\
\hline Amilasa urinaria & $19 \mathrm{UI} / \mathrm{L}$ \\
\hline Amilasa sérica & $1174 \mathrm{UI} / \mathrm{L}$ \\
\hline Creatinina & $0,5 \mathrm{mg} / \mathrm{dL}$ \\
\hline Creatinina orina 24 horas & $43,9 \mathrm{mg} / \mathrm{dL}$ \\
\hline Macroamilasemia* & Positivo \\
\hline
\end{tabular}

${ }^{*}$ Cromatografía; precipitación con polietilenglicol (PEG). Fuente: Sistema de Atención Historia Clínica Hospital Lazarte Echegaray - Essalud. Depuración de amilasa(VN: $<5)$; amilasa urinaria (VN: $<460 \mathrm{U} / \mathrm{L}$ ); amilasa sérica (VN: 0-100 U/L); creatinina sérica (VN: 0,4-1,4 mg/dL)

milasemia, lo que llevó al diagnóstico equivocado de pancreatitis aguda; de las causas de hiperamilasemia, del 6\% al 9,6\% se deben a una macroamilasemia $(14,15)$. Lo más destacado de nuestro caso es que la evolución de la hiperamilasemia fue sin dolor abdominal, por lo que se realizaron los estudios pertinentes para descartar otras causas pancreáticas y extrapancreáticas, así como las asociaciones de otras enfermedades. La enfermedad celíaca es la patología asociada con mayor frecuencia; en un estudio de casos y controles llevado a cabo por Rabsztyn y colaboradores (16), los autores concluyeron que un porcentaje significativo de los pacientes recién diagnosticados con enfermedad celíaca tienen macroamilasemia, por lo que, en presencia de esta, se debe considerar la posibilidad de enfermedad celíaca. En nuestro caso, se realizó endoscopia con toma de biopsia con 6 muestras y se encontró un MARSH 0, sin criterio para ampliar el estudio de enfermedad celíaca. En cuanto a las otras patologías asociadas están las enfermedades hepáticas crónicas (17), la diabetes mellitus (18), la artritis reumatoide (19), el lupus eritematoso sistémico (20), el linfoma de células T (21), la enfermedad inflamatoria intestinal (22), el VIH asintomático (23) y el carcinoma de células renales y tiroides (16). Todas estas patologías fueron descartadas en nuestra paciente.

Levitt y colaboradores propusieron la existencia de dos formas de macroamilasemia (24): una forma se caracterizó por la presencia de malabsorción con una amilasa sérica 11 $S$ resultante de la unión de amilasa de tamaño normal por inmunoglobulina IgA. En la otra forma de macroamilasemia, los pacientes no sufrieron malabsorción, el coeficiente de sedimentación fue de $7 \mathrm{~S}$ y el complejo no fue el resultado de

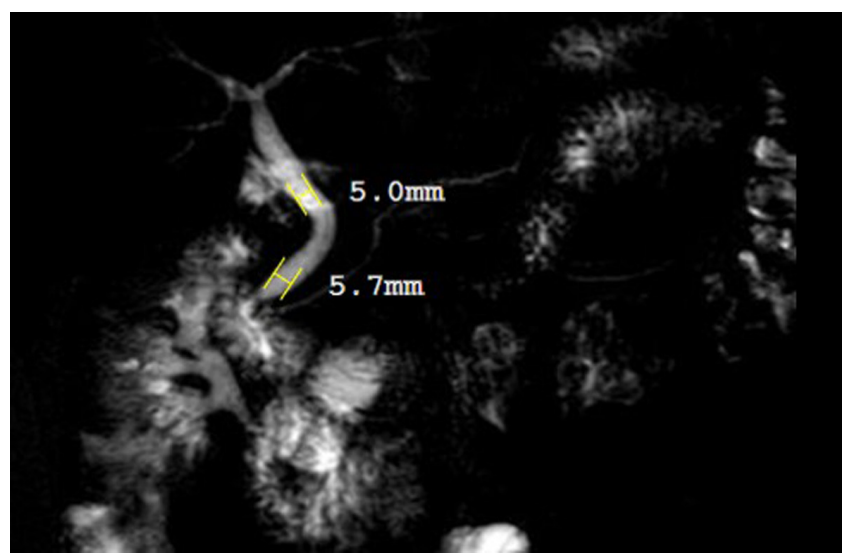

Figura 2. Colangiorresonancia.

la unión de la amilasa a una inmunoglobulina. Actualmente se describe 3 tipo de macroamilasemia (Tabla 3) (25).

Tabla 3. Tipos de macroamilasemia

\begin{tabular}{llll|}
\multicolumn{1}{c}{ Tipo } & \multicolumn{1}{c}{1} & \multicolumn{1}{c|}{$\mathbf{2}$} & \multicolumn{1}{c|}{3} \\
\hline Amilasa sérica & $\begin{array}{l}\text { Amilasa elevada } \\
\text { persistente }\end{array}$ & Aumentada & Normal \\
Amilasa urinaria & Disminuida & $\begin{array}{l}\text { Disminuida o } \\
\text { normal }\end{array}$ & Normal \\
ACR & Muy disminuido & Disminuido & Disminuido \\
$\begin{array}{l}\text { Concentración de } \\
\text { macroamilasa }\end{array}$ & $\begin{array}{l}\text { Relativamente } \\
\text { alto }\end{array}$ & $\begin{array}{l}\text { Menor que el } \\
\text { tipo 1 }\end{array}$ & Baja \\
Tiempo de resultado & Breve & Largos & Largos \\
\hline
\end{tabular}

Fuente: tomado de (25).

La paciente presentó una depuración de amilasa (ACR) muy disminuida $(0,02 \%)$ y el tiempo de resultado positivo fue breve, correspondiente a una macroamilasemia tipo 1 , que fue confirmada con cromatografía, de precipitación con polietilenglicol (PEG). Se puede concluir que este caso presentado corresponde a una macroamilasemia tipo 1. La macroamilasemia debe ser considerada en el caso de una hiperamilasemia persistente, incluso si este cuadro debutó con dolor abdominal, descartando también otras etiologías y asociaciones de importancia.

\section{Conflicto de intereses}

El autor declara no tener ningún conflicto de intereses. 


\section{REFERENCIAS}

1. Banks PA, Bollen TL, Dervenis Ch, Gooszeng G, Johnson $\mathrm{CD}$, Sarr MG, et al. Classification of acute pancreatitis-2012: Revision of Atlanta clasification and definitions by international consensus. Gut. 2013;62:102-11. https://doi.org/10.1136/gutjnl-2012-302779

2. Yokoe M, Takada T, Mayumi T, Yoshida M, Isaji S, Wada $\mathrm{K}$, et al. Japanese guidelines for the management of acute pancreatitis: Japanese Guidelines 2015. J Hepatobiliary Pancreat Sci. 2015;22:405-32. https://doi.org/10.1002/jhbp.259

3. Crockett SD, Wani S, Gardner T, Falck-Ytter Y, Barkun A, et al. American Gastroenterological Association Institute Guideline on Initial Management of Acute Pancreatitis. Gastroenterology. 2018;154(4):1096-101. https://doi.org/10.1053/j.gastro.2018.01.032

4. Greenberg JA, Hsu J, Bawazeer M, Marshall J, Friedrich J, Nathens A, et al. Clinical practice guideline: management of acute pancreatitis. Can J Surg. 2016;59(2):128-40. https://doi.org/10.1503/cjs.015015

5. Wiederkehr JC, Wiederkehr B, Wiederkehr E, Carvalho C. Nonspecific Hyperamylasemia: A Case Report. JOP. 2013;14(1):74-6. https://doi.org/10.6092/1590-8577/1216

6. Sánchez-Molina Acosta MI, Chueca MP, Quílez D, Vergara E. ¿Macroamilasemia o hiperamilasemia en un paciente con dolor abdominal? Rev Lab Clin. 2016;9(1):13-6. https://doi.org/10.1016/j.labcli.2015.08.003

7. Pezzilli R, Andreone P, Morselli-Labate AM, Sama C, Billi P, Cursaro C, et al. Serum pancreatic enzyme concentrations in chronic viral liver diseases. Dig Dis Sci. 1999;44:350-5. https://doi.org/10.1023/A:1026662719514

8. Antonini F, Pezzilli R, Angelelli L, Macarri G. Pancreatic disorders in inflammatory bowel disease. World J Gastrointest Pathophysiol. 2016;7(3):276-82. https://doi.org/10.4291/wjgp.v7.i3.276

9. Berk JE, Kizu H, Wilding P, Searcy RL. Macroamylasemia: a newly recognized cause for elevated serum amylase activity. N Engl J Med. 1967;277:941-6. https://doi.org/10.1056/NEJM196711022771801

10. Šimac V, Špelić M, Devčić B, Rački S. Diagnosing macroamylasemia in unexplained hyperamylasemia. Acta Med Croatica. 2017;71(1)63-7.

11. Oette M. Macroamylasemia. En: Lang F (editor). Encyclopedia of Molecular Mechanisms of Disease. Berlín, Springer: 2009. p. 1241-1242.

12. Vahedi A, Mehramouz B, Maroufi P, Pourlak T, Rezvanpour $\mathrm{R}$, Shokouhi B, et al. Determining the Frequency of Macroamylasemia in Patients with Hyperamylasemia using PEG Precipitation Method. J Clin Diag Res. 2018;12(5):EC10-EC13. https://doi.org/10.7860/JCDR/2018/34148.11466
13. Levitt MD, Ellis C. A rapid and simple assay to determine if macroamylase is the cause of hyperamylasemia. Gastroenterol. 1982;83(2):378-82. https://doi.org/10.1016/S0016-5085(82)80331-4

14. Forsman RW. Macroamylase: Prevalence, distribution of age, sex, amylase activity, and electrophoretic mobility. Clin Biochem. 1986;19(4):250-3. https://doi.org/10.1016/S0009-9120(86)80036-4

15. Warshaw AL, Hawboldt MM. Puzzling persistent hyperamylasemia, probably neither pancreatic nor pathologic. Am J Surg. 1988;155(3):453-6. https://doi.org/10.1016/S0002-9610(88)80112-0

16. Rabsztyn A, Green P, Berti I, Fasano A, Perman J, Karoly H. Macroamylasemia in Patients With Celiac Disease. Am J Gastroenterol. 2001;96(4):1096-100. https://doi.org/10.1111/j.1572-0241.2001.03746.x

17. Yoshida E, Tsuruoka T, Suzuki M, Asahara M, Okazaki T, Kadohno N, et al. [Sex and age distribution of patients with macroamylasemia found in the daily isoenzyme analysis]. Rinsho Byori. 1998;46(5):473-8.

18. Masuda H, Tsukada T, Nakayama M, Kitamura M. A study on 41-cases of macroamylasemia detected in a period of 15 months. Chemistry. 1983;27:201-7.

19. Cutolo M, Sulli A, Barone A, Picciotto A, Mangravtti S, Seriolo B, et al. Macroamylasemia: a possible cause of unexplained hyperamylasemia in rheumatoid arthritis. Br J Rheumatol. 1995;34(3):290-2. https://doi.org/10.1093/rheumatology/34.3.290

20. Goto H, Wakui H, Komatsuda A, Imai H, Miura A, Fujita K, et al. Simultaneous Macroamylasemia and Macrolipasemia in a Patient with Systemic Lupus Erythematosus in Remission. Intern Med. 2000;39(12):1115-8. https://doi.org/10.2169/internalmedicine.39.1115

21. Okada S, Komatsu N, Osaka A, Yoshida M, Sakamoto S, Miura Y, et al. IgG (kappa type) binding macroamylasemia complicated with immunoblastic lymphadenopathylike T cell lymphoma. Nippon Naika Gakkai Zasshi. 1989;78(4):519-22. https://doi.org/10.2169/naika.78.519

22. Fujimura $Y$, Nishishita $C$, Uchida J, Iida $M$. Macroamylasemia associated with ulcerative colitis. J Mol Med. 1995;73:95-7. https://doi.org/10.1007/BF00270584

23. Eleccion C, Hathaway A. Macroamylasemia in HIV infection. Tex Med. 1998;94(12):77-9.

24. Levitt MD, Goetzl EJ, Cooperbani SR. Two forms of macroamylasemia. Lancet. 1968;291(7549):95758. https://doi.org/10.1016/S0140-6736(68)90907-0

25. Fridhandler, Berk E. Macroamylasemia. Advances Clin Chem. 1978;20:267-86. https://doi.org/10.1016/S0065-2423(08)60024-4 